\title{
Tallness and Level by Level Equivalence and Inequivalence ${ }^{* \dagger}$
}

\author{
Arthur W. Apter $\$ \S$ \\ Department of Mathematics \\ Baruch College of CUNY \\ New York, New York 10010 USA \\ and \\ The CUNY Graduate Center, Mathematics \\ 365 Fifth Avenue \\ New York, New York 10016 USA \\ http://faculty.baruch.cuny.edu/apter \\ awapter@alum.mit.edu \\ September 7, 2008 \\ (revised April 3, 2009)
}

\begin{abstract}
We construct two models containing exactly one supercompact cardinal in which all nonsupercompact measurable cardinals are strictly taller than they are either strongly compact or supercompact. In the first of these models, level by level equivalence between strong compactness and supercompactness holds. In the other, level by level inequivalence between strong compactness and supercompactness holds. Each universe has only one strongly compact cardinal and contains relatively few large cardinals.
\end{abstract}

\section{Introduction and Preliminaries}

We begin with definitions due to Hamkins which are found in [9]. For $\kappa$ a measurable cardinal and $\alpha$ any ordinal, $\kappa$ is $\alpha$ tall if there is an elementary embedding $j: V \rightarrow M$ having critical point $\kappa$ such that $j(\kappa)>\alpha$ and $M^{\kappa} \subseteq M . \kappa$ is tall if $\kappa$ is $\alpha$ tall for every ordinal $\alpha$. In [9], Hamkins

*2000 Mathematics Subject Classifications: 03E35, 03E55.

${ }^{\dagger}$ Keywords: Supercompact cardinal, strongly compact cardinal, superstrong cardinal, strong cardinal, tall cardinal, level by level equivalence between strong compactness and supercompactness, level by level inequivalence between strong compactness and supercompactness.

¥The author's research was partially supported by PSC-CUNY grants and CUNY Collaborative Incentive grants.

$\S$ The author wishes to thank the referee, for helpful comments and suggestions which have been incorporated into the current version of the paper. 
began the study of tall cardinals and showed that they satisfy many interesting and fundamental properties. In particular, the existence of any number of tall cardinals (one tall cardinal, $\gamma$ many tall cardinals for $\gamma$ an arbitrary ordinal, or even a proper class of tall cardinals) is equiconsistent with the same number of strong cardinals, thereby showing the nontriviality of tallness. Further, in analogy to strongness, $\alpha$ tallness is witnessed by an extender embedding (which, without loss of generality, may be assumed to have rank below the least strong limit cardinal above $\alpha$ ), and if $\delta$ is $\alpha$ tall, then $\delta$ is $\beta$ tall for every $\beta<\alpha$.

We will extend Hamkins' study of tallness to the context of level by level equivalence between strong compactness and supercompactness and level by level inequivalence between strong compactness and supercompactness. Before doing so, we first present a few additional relevant definitions. For any ordinal $\delta$, let $\sigma_{\delta}$ be the smallest measurable cardinal greater than $\delta$. For any non-supercompact measurable cardinal $\delta$, let $\theta_{\delta}$ be the smallest cardinal greater than $\delta$ such that $\delta$ is not $\theta_{\delta}$ supercompact. For any non-strongly compact measurable cardinal $\delta$, let $\rho_{\delta}$ be the smallest cardinal greater than $\delta$ such that $\delta$ is not $\rho_{\delta}$ strongly compact. Observe that if $\delta$ is not strongly compact, then both $\theta_{\delta}$ and $\rho_{\delta}$ are defined, and $\theta_{\delta} \leq \rho_{\delta}$.

Suppose $V$ is a model of ZFC in which for all regular cardinals $\delta<\lambda, \delta$ is $\lambda$ strongly compact iff $\delta$ is $\lambda$ supercompact. Such a universe will be said to witness level by level equivalence between strong compactness and supercompactness. Suppose now that $V$ is a model of ZFC in which if $\delta$ is measurable but not supercompact, then $\delta$ is $\theta_{\delta}$ strongly compact. $V$ is then said to witness level by level inequivalence between strong compactness and supercompactness. A non-supercompact measurable cardinal $\delta$ which is $\theta_{\delta}$ strongly compact is said to witness level by level inequivalence between strong compactness and supercompactness. For brevity, in all of these definitions, we will henceforth eliminate the phrase "between strong compactness and supercompactness."

Models containing supercompact cardinals in which level by level equivalence holds were first constructed in [4]. A model with exactly one supercompact cardinal in which level by level inequivalence holds was constructed in [1]. A theorem of Magidor (see [4, Lemma 7]) shows that if $\kappa$ is supercompact, then there are always cardinals $\delta<\lambda<\kappa$ such that $\lambda$ is singular of cofinality 
greater than or equal to $\delta, \delta$ is $\lambda$ strongly compact, but $\delta$ is not $\lambda$ supercompact. As a consequence of Magidor's theorem, it follows that if $V \vDash$ "Level by level equivalence holds and $\delta$ is measurable but not strongly compact", then either $\rho_{\delta}=\theta_{\delta}$ or $\rho_{\delta}=\left(\theta_{\delta}\right)^{+}$.

The purpose of this paper is to establish two results which show that it is possible to have models for level by level equivalence and level by level inequivalence containing exactly one supercompact cardinal and no other strongly compact cardinals in which each non-supercompact measurable cardinal $\delta$ is strictly taller than it is either strongly compact or supercompact. To avoid trivialities, our witnesses for tallness for $\delta$ will be inaccessible cardinals greater than $\rho_{\delta} .{ }^{1}$ Specifically, we prove the following two theorems.

Theorem 1 Suppose $V \vDash " Z F C+\kappa$ is supercompact". There is then a partial ordering $\mathbb{P} \subseteq V$ such that $V^{\mathbb{P}} \vDash " Z F C+G C H+\kappa$ is supercompact + No cardinal is supercompact up to a measurable cardinal". In $V^{\mathbb{P}}$, level by level equivalence holds, and $\kappa$ is the only strongly compact cardinal. Further, in $V^{\mathbb{P}}$, every non-supercompact measurable cardinal $\delta$ is $\lambda_{\delta}$ tall, for some inaccessible cardinal $\lambda_{\delta}>\rho_{\delta}$. In particular, in $V^{\mathbb{P}}$, every non-supercompact measurable cardinal $\delta$ is taller than it is strongly compact.

Theorem 2 Suppose $V \vDash " Z F C+G C H+\kappa<\lambda$ are such that $\kappa$ is $\lambda$ supercompact and $\lambda$ is the least inaccessible cardinal above $\kappa+$ Level by level equivalence holds". There is then a partial ordering $\mathbb{P} \in V$, a submodel $\bar{V} \subseteq V^{\mathbb{P}}$, and a cardinal $\kappa_{0}<\kappa$ such that $\bar{V} \vDash " Z F C+G C H+$ $\kappa_{0}$ is supercompact + No cardinal is supercompact up to an inaccessible cardinal". In $\bar{V}$, level by level inequivalence holds, and $\kappa_{0}$ is the only strongly compact cardinal. Further, in $\bar{V}$, every nonsupercompact measurable cardinal $\delta$ is $\lambda_{\delta}$ tall, for some inaccessible cardinal $\lambda_{\delta}>\rho_{\delta}$. In particular, in $\bar{V}$, every non-supercompact measurable cardinal $\delta$ is taller than it is strongly compact.

\footnotetext{
${ }^{1}$ Hamkins has shown in [9, Lemma 2.1] that if $\delta$ is $\lambda$ tall, then $\delta$ is $(\lambda)^{\delta}$ tall. He has further shown in [9, Theorem $2.11]$ that if $\delta$ is $\lambda$ strongly compact, then $\delta$ is $\lambda^{+}$tall. Thus, any (fully) strongly compact cardinal must be tall. However, as the referee has pointed out, it is possible to infer from [9, Theorem 2.11] that if $\delta$ is measurable and $\rho_{\delta}=\lambda^{+}$, then $\delta$ is strictly taller than it is strongly compact. This is since $\delta$ is (at least) $\lambda^{+}$tall, yet $\delta$ is only $\lambda$ strongly compact. If, on the other hand, $\rho_{\delta}$ is a limit cardinal, then the proof given in [9, Theorem 2.11] will not allow us to conclude that $\delta$ is strictly taller than it is strongly compact. This is because Hamkins' argument uses that a $\lambda$ strong compactness embedding actually witnesses $\lambda^{+}$tallness, so when $\rho_{\delta}$ is a limit cardinal, it is unclear how to find one strong compactness embedding which gives enough tallness. Whether or not there is a ZFC proof that every non-strongly compact measurable cardinal is strictly taller than it is strongly compact is an open question.
} 
In fact, for both Theorems 1 and 2, the witnessing models will be such that every measurable cardinal $\delta$ is $\lambda_{\delta}$ tall, where $\lambda_{\delta}$ is the least weakly compact cardinal above $\rho_{\delta}$, the least Ramsey cardinal above $\rho_{\delta}$, the least Ramsey limit of Ramsey cardinals above $\rho_{\delta}$, or in general, where $\lambda_{\delta}$ is any "reasonable" large cardinal provably below the least measurable cardinal above $\rho_{\delta}$. We will explain this in greater detail towards the end of the paper.

We now very briefly give some preliminary information concerning notation and terminology. When forcing, $q \geq p$ means that $q$ is stronger than $p$. If $G$ is $V$-generic over $\mathbb{P}$, we will abuse notation slightly and use both $V[G]$ and $V^{\mathbb{P}}$ to indicate the universe obtained by forcing with $\mathbb{P}$. We will, from time to time, confuse terms with the sets they denote and write $x$ when we actually mean $\dot{x}$ or $\check{x}$.

The partial ordering $\mathbb{P}$ is $\kappa$-strategically closed if in the two person game in which the players construct an increasing sequence $\left\langle p_{\alpha}: \alpha \leq \kappa\right\rangle$, where player I plays odd stages and player II plays even stages (choosing the trivial condition at stage 0), player II has a strategy which ensures the game can always be continued. $\mathbb{P}$ is $\prec \kappa$-strategically closed if in the two person game in which the players construct an increasing sequence $\left\langle p_{\alpha}: \alpha<\kappa\right\rangle$, where player I plays odd stages and player II plays even stages (choosing the trivial condition at stage 0), player II has a strategy which ensures the game can always be continued. $\mathbb{P}$ is $(\kappa, \infty)$-distributive if given a sequence $\left\langle D_{\alpha}: \alpha<\kappa\right\rangle$ of dense open subsets of $\mathbb{P}, \bigcap_{\alpha<\kappa} D_{\alpha}$ is dense open as well. Note that if $\mathbb{P}$ is $\kappa$-strategically closed, then $\mathbb{P}$ is $(\kappa, \infty)$-distributive. Further, if $\mathbb{P}$ is $(\kappa, \infty)$-distributive and $f: \kappa \rightarrow V$ is a function in $V^{\mathbb{P}}$, then $f \in V$.

A corollary of Hamkins' work on gap forcing found in [7] and [8] will be employed in the proof of Theorem 2. We therefore state as a separate theorem what is relevant for this paper, along with some associated terminology, quoting from [7] and [8] when appropriate. Suppose $\mathbb{P}$ is a partial ordering which can be written as $\mathbb{Q} * \dot{\mathbb{R}}$, where $|\mathbb{Q}|<\delta, \mathbb{Q}$ is nontrivial, and $\vdash_{\mathbb{Q}}$ " $\mathbb{R}$ is $\delta$-strategically closed". In Hamkins' terminology of [7] and [8], $\mathbb{P}$ admits a gap at $\delta$. In Hamkins' terminology of [7] and [8], $\mathbb{P}$ is mild with respect to a cardinal $\kappa$ iff every set of ordinals $x$ in $V^{\mathbb{P}}$ of size less than $\kappa$ has a "nice" name $\tau$ in $V$ of size less than $\kappa$, i.e., there is a set $y$ in $V,|y|<\kappa$, such that any 
ordinal forced by a condition in $\mathbb{P}$ to be in $\tau$ is an element of $y$. Also, as in the terminology of [7], [8], and elsewhere, an embedding $j: \bar{V} \rightarrow \bar{M}$ is amenable to $\bar{V}$ when $j \uparrow A \in \bar{V}$ for any $A \in \bar{V}$. The specific corollary of Hamkins' work from [7] and [8] we will be using is then the following.

Theorem 3 (Hamkins) Suppose that $V[G]$ is a generic extension obtained by forcing that admits a gap at some regular $\delta<\kappa$. Suppose further that $j: V[G] \rightarrow M[j(G)]$ is an embedding with critical point $\kappa$ for which $M[j(G)] \subseteq V[G]$ and $M[j(G)]^{\delta} \subseteq M[j(G)]$ in $V[G]$. Then $M \subseteq V$; indeed, $M=V \cap M[j(G)]$. If the full embedding $j$ is amenable to $V[G]$, then the restricted embedding $j \uparrow V: V \rightarrow M$ is amenable to $V$. If $j$ is definable from parameters (such as a measure or extender) in $V[G]$, then the restricted embedding $j \uparrow V$ is definable from the names of those parameters in $V$. Finally, if $\mathbb{P}$ is mild with respect to $\kappa$ and $\kappa$ is $\lambda$ strongly compact in $V[G]$ for any $\lambda \geq \kappa$, then $\kappa$ is $\lambda$ strongly compact in $V$.

We assume familiarity with the large cardinal notions of measurability, strongness, superstrongness, strong compactness, and supercompactness. Readers are urged to consult [10] for further details. Note that we will say that $\kappa$ is supercompact (or strongly compact or tall) up to the cardinal $\lambda$ if $\kappa$ is $\gamma$ supercompact (or $\gamma$ strongly compact or $\gamma$ tall) for every $\gamma<\lambda$.

\section{The Proofs of Theorems 1 and 2}

We turn now to the proof of Theorem 1.

Proof: Suppose $V \vDash$ "ZFC $+\kappa$ is supercompact". By first forcing GCH, then forcing with the partial ordering of [4], and then taking the appropriate submodel if necessary, we slightly abuse notation and assume in addition that $V \vDash$ "ZFC $+\mathrm{GCH}+\kappa$ is supercompact + No cardinal is supercompact up to a measurable cardinal + Level by level equivalence holds". In particular, $V \vDash " \kappa$ is the only strongly compact cardinal".

We are now in a position to define the partial ordering $\mathbb{P}$ used in the proof of Theorem 1. Let $A=\{\delta<\kappa: \delta$ is a non-superstrong measurable cardinal $\}$. $\mathbb{P}$ is the reverse Easton iteration of length $\kappa$ which begins by adding a Cohen subset of $\omega$ and then does nontrivial forcing only at 
stages $\delta \in A$, where it adds a non-reflecting stationary set of ordinals of cofinality $\omega$ to $\delta .^{2}$ Standard arguments show that $V^{\mathbb{P}} \vDash \mathrm{GCH}$ and that $V$ and $V^{\mathbb{P}}$ have the same cardinals and cofinalities.

Lemma 2.1 Suppose $\delta<\lambda$ are both regular cardinals and $\delta<\kappa$. If $V \vDash " \delta$ is $\lambda$ supercompact and $\delta$ is superstrong", then $V^{\mathbb{P}} \vDash$ " $\delta$ is $\lambda$ supercompact".

Proof: Let $j: V \rightarrow M$ be an elementary embedding witnessing the $\lambda$ supercompactness of $\delta$ generated by a supercompact ultrafilter over $P_{\delta}(\lambda)$. Write $\mathbb{P}=\mathbb{P}_{\delta} * \dot{\mathbb{P}}^{\delta}$. Since $V \vDash$ "No cardinal is supercompact up to a measurable cardinal", $\lambda<\sigma_{\delta}$. Therefore, by the definition of $\mathbb{P}$, $\Vdash_{\mathbb{P}_{\delta}}$ " $\dot{\mathbb{P}}^{\delta}$ is $\prec\left(\sigma_{\delta}\right)^{V}$-strategically closed". In addition, because $\left|\mathbb{P}_{\delta}\right|=\delta$, by the Lévy-Solovay results [11], it is the case that $\vdash_{\mathbb{P}_{\delta}}$ " $\left(\sigma_{\delta}\right)^{V}=\left(\sigma_{\delta}\right)^{V^{\mathbb{P}} \delta}$. Thus, to show that $V^{\mathbb{P}} \vDash " \delta$ is $\lambda$ supercompact", it suffices to show that $V^{\mathbb{P}_{\delta}} \vDash " \delta$ is $\lambda$ supercompact". However, by the facts that $\lambda<\sigma_{\delta}$ and $M^{\lambda} \subseteq M, M \vDash$ "No cardinal in the half-open interval $(\delta, \lambda]$ is measurable". Further, since by GCH, $\lambda \geq \delta^{+}=2^{\delta}$, as in [2, Lemma 2.1], $M \vDash " \delta$ is superstrong". This means that $j\left(\mathbb{P}_{\delta}\right)=\mathbb{P}_{\delta} * \dot{\mathbb{Q}}$, where the first ordinal at which $\dot{\mathbb{Q}}$ is forced to do nontrivial forcing is well above $\lambda$. A standard argument now shows that $j$ lifts in $V^{\mathbb{P}_{\delta}}$ to a $\lambda$ supercompactness embedding $j: V^{\mathbb{P}_{\delta}} \rightarrow M^{j\left(\mathbb{P}_{\delta}\right)}{ }^{3}$ This completes the proof of Lemma 2.1.

Suppose $\lambda>\kappa$ is regular. Let $j: V \rightarrow M$ be an elementary embedding witnessing the $\lambda$ supercompactness of $\kappa$ generated by a supercompact ultrafilter over $P_{\kappa}(\lambda)$. Since $V \vDash$ "No cardinal is supercompact up to a measurable cardinal", $V \vDash$ "There are no measurable cardinals above $\kappa$ ". Thus, $V \vDash$ " $\lambda$ is not measurable". The proof of Lemma 2.1 consequently shows that $j$ lifts in $V^{\mathbb{P}}$ as before to $j: V^{\mathbb{P}} \rightarrow M^{j(\mathbb{P})}$. It therefore follows as an immediate corollary of the proof of Lemma 2.1 that $V^{\mathbb{P}} \vDash " \kappa$ is supercompact".

\footnotetext{
${ }^{2}$ The precise definition of this partial ordering may be found in either [4] or [2]. We do wish to note here, however, that it is $\prec \delta$-strategically closed. Also, whenever $\delta$ is inaccessible, this partial ordering has cardinality $\delta$.

${ }^{3}$ An outline of this argument is as follows. Let $G_{0}$ be $V$-generic over $\mathbb{P}_{\delta}$. The same method as found, e.g., in the construction of the generic object $G_{1}$ in the proof of [2, Lemma 2.4] now allows us to build in $V\left[G_{0}\right]$ an $M\left[G_{0}\right]$-generic object $G_{1}$ over $\mathbb{Q}$. This argument uses that by $\mathrm{GCH}$ and the fact that $j$ is given by an ultrapower embedding, we may let $\left\langle D_{\alpha}: \alpha<\lambda^{+}\right\rangle$enumerate in $V\left[G_{0}\right]$ the dense open subsets of $\mathbb{Q}$ present in $M\left[G_{0}\right]$. Because $M\left[G_{0}\right]$ remains $\lambda$-closed with respect to $V\left[G_{0}\right]$, by the $\prec \lambda^{+}$-strategic closure of $\mathbb{Q}$ in both $M\left[G_{0}\right]$ and $V\left[G_{0}\right]$, we may work in $V\left[G_{0}\right]$ and meet each $D_{\alpha}$ in order to construct $G_{1}$. Since $j^{\prime \prime} G_{0} \subseteq G_{0} * G_{1}$, we may lift $j$ in $V\left[G_{0}\right]$ to $j: V\left[G_{0}\right] \rightarrow M\left[G_{0}\right]\left[G_{1}\right]$.
} 
Lemma $2.2 V^{\mathbb{P}} \vDash$ "No cardinal is supercompact up to a measurable cardinal".

Proof: Suppose $\delta \leq \lambda$ are such that $V^{\mathbb{P}} \vDash$ " $\delta$ is $\alpha$ supercompact for every $\alpha<\lambda$ and $\lambda$ is measurable". By its definition, we may write $\mathbb{P}=\mathbb{P}^{\prime} * \dot{\mathbb{P}}^{\prime \prime}$, where $\left|\mathbb{P}^{\prime}\right|=\omega, \mathbb{P}^{\prime}$ is nontrivial, and $\Vdash_{\mathbb{P}^{\prime}}$ " $\dot{P}^{\prime \prime}$ is $\aleph_{1}$-strategically closed". Therefore, by Theorem 3 , it must be the case that $V \vDash$ " $\delta$ is $\alpha$ supercompact for every $\alpha<\lambda$ and $\lambda$ is measurable" as well. Since this is contradictory to our hypotheses about $V$, this completes the proof of Lemma 2.2 .

Lemma 2.3 $V^{\mathbb{P}} \vDash$ "Level by level equivalence holds".

Proof: Suppose $\delta \leq \lambda$ are regular cardinals such that $V^{\mathbb{P}} \vDash$ " $\delta$ is $\lambda$ strongly compact". As has already been observed, $V \vDash$ "There are no measurable cardinals above $\kappa$ ". Consequently, because $|\mathbb{P}|=\kappa$, the results of [11] allow us to infer that $V^{\mathbb{P}} \vDash$ "There are no measurable cardinals above $\kappa$ ". Hence, because $V^{\mathbb{P}} \vDash$ " $\kappa$ is supercompact", we may assume without loss of generality that $\delta<\kappa$.

Note that from its definition, $\mathbb{P}$ is mild with respect to $\delta$. Thus, by the factorization of $\mathbb{P}$ (as $\left.\mathbb{P}^{\prime} * \dot{\mathbb{P}}^{\prime \prime}\right)$ given in Lemma 2.2 and Theorem $3, V \vDash$ " $\delta$ is $\lambda$ strongly compact". Since level by level equivalence holds in $V, V \vDash$ " $\delta$ is $\lambda$ supercompact" as well. Further, it must be the case that $V \vDash$ " $\delta$ is superstrong". (If not, then by the definition of $\mathbb{P}, V^{\mathbb{P}} \vDash$ " $\delta$ contains a non-reflecting stationary set of ordinals of cofinality $\omega$ and thus is not weakly compact".) Therefore, by Lemma $2.1, V^{\mathbb{P}} \vDash$ " $\delta$ is $\lambda$ supercompact". This completes the proof of Lemma 2.3.

Lemma 2.4 $V^{\mathbb{P}} \vDash$ "Every measurable cardinal $\delta$ is tall up to $\left(\sigma_{\delta}\right)^{V}$ ".

Proof: Let $\delta \neq \kappa$ be such that $V^{\mathbb{P}} \vDash " \delta$ is measurable". As in the proof of Lemma 2.3, we may assume without loss of generality that $\delta<\kappa$ and that $V \vDash$ " $\delta$ is superstrong". Let $\lambda<\left(\sigma_{\delta}\right)^{V}$ be an arbitrary inaccessible cardinal which is not a limit of inaccessible cardinals. By the factorization of 
$\mathbb{P}\left(\right.$ as $\left.\mathbb{P}_{\delta} * \dot{\mathbb{P}}^{\delta}\right)$ given in Lemma 2.1, to show that $V^{\mathbb{P}} \vDash " \delta$ is $\lambda$ tall", it suffices to show that $V^{\mathbb{P}_{\delta}} \vDash " \delta$ is $\lambda$ tall".

To do this, we first observe that because $V \vDash$ " $\delta$ is superstrong", $V \vDash$ " $\delta$ is $\lambda$ strong". (This follows since an easy reflection argument shows that any superstrong cardinal is strong well beyond the least measurable cardinal above it.) Using this fact, we will now argue in analogy to the proof of [9, Theorem 4.1] (which itself uses ideas originally due to Magidor that are found in the proofs of [2, Lemma 2.4] and [3, Lemma 2.3], as well as elsewhere in the literature), quoting freely (or perhaps verbatim) from the relevant arguments when appropriate. Let $j: V \rightarrow M$ be an elementary embedding witnessing the $\lambda$ strongness of $\delta$ generated by a $(\delta, \lambda)$-extender. We may assume without loss of generality that $M^{\delta} \subseteq M$ and that there is no $(\delta, \lambda)$-extender $\mathcal{F} \in M$ (so that in particular, $M \vDash$ " $\delta$ is measurable but not superstrong"). The cardinal $\lambda$ is large enough so that we may choose a normal measure $\mathcal{U} \in M$ over $\delta$ having trivial Mitchell rank and let $k: M \rightarrow N$ be the elementary embedding generated by $\mathcal{U}$. As in [9, Theorem 4.1], $i=k \circ j$ is an elementary embedding witnessing the $\lambda$ tallness of $\delta$. We show that $i: V \rightarrow N$ lifts in $V^{\mathbb{P}_{\delta}}$ to $i: V^{\mathbb{P}_{\delta}} \rightarrow N^{i\left(\mathbb{P}_{\delta}\right)}$. Because this lifted embedding will witness the $\lambda$ tallness of $\delta$ in $V^{\mathbb{P}_{\delta}}$ and $\lambda$ was arbitrary, this will prove Lemma 2.4.

Let $G_{0}$ be $V$-generic over $\mathbb{P}_{\delta}$. Since $N \vDash$ " $\delta$ is not measurable", only trivial forcing is done at stage $\delta$ in $N^{\mathbb{P}_{\delta}}$ in the definition of $i\left(\mathbb{P}_{\delta}\right)$. Thus, we may write $i\left(\mathbb{P}_{\delta}\right)=\mathbb{P}_{\delta} * \dot{\mathbb{Q}}^{1} * \dot{\mathbb{Q}}^{2}$, where $\dot{\mathbb{Q}}^{1}$ is forced to do nontrivial forcing at ordinals in the half-open interval $(\delta, k(\delta)]$, and $\dot{\mathbb{Q}}^{2}$ is forced to do nontrivial forcing at ordinals in the open interval $(k(\delta), k(j(\delta)))$, i.e., at ordinals in the open interval $(k(\delta), i(\delta))$. We will build in $V\left[G_{0}\right]$ generic objects $G_{1}$ and $G_{2}$ for $\mathbb{Q}^{1}$ and $\mathbb{Q}^{2}$ respectively. As it will be the case that $i^{\prime \prime} G_{0} \subseteq G_{0} * G_{1} * G_{2}, i$ will lift in $V\left[G_{0}\right]$ to $i: V\left[G_{0}\right] \rightarrow N\left[G_{0}\right]\left[G_{1}\right]\left[G_{2}\right]$, and the proof of Lemma 2.4 will be complete.

To construct $G_{1}$, note that since $k$ is generated by the ultrafilter $\mathcal{U}$ over $\delta$, by GCH in $V, M$, and $N, V \vDash "\left|k\left(\delta^{+}\right)\right|=\left|k\left(2^{\delta}\right)\right|=\left|\left\{f: f: \delta \rightarrow \delta^{+}\right\}\right|=\left|\left[\delta^{+}\right]^{\delta}\right|=\delta^{+}$". Thus, as $N\left[G_{0}\right] \vDash "\left|\wp\left(\mathbb{Q}^{1}\right)\right|=$ $\left|k\left(2^{\delta}\right)\right|$ ", we can let $\left\langle D_{\alpha}: \alpha<\delta^{+}\right\rangle \in V\left[G_{0}\right]$ enumerate the dense open subsets of $\mathbb{Q}^{1}$ present in $N\left[G_{0}\right]$. Using the fact that $\mathbb{Q}^{1}$ is $\prec \delta^{+}$-strategically closed in $N\left[G_{0}\right], M\left[G_{0}\right]$, and $V\left[G_{0}\right]$, the same argument as given in the construction of the generic object $G_{1}$ in the proof of [2, Lemma 2.4] is 
once again applicable and allows us to build in $V\left[G_{0}\right]$ an $N\left[G_{0}\right]$-generic object $G_{1}$ over $\mathbb{Q}^{1}$.

To construct $G_{2}$, we first write $j\left(\mathbb{P}_{\delta}\right)=\mathbb{P}_{\delta} * \dot{\mathbb{R}}^{1} * \dot{\mathbb{R}}^{2}$. Since $M \vDash$ " $\delta$ is measurable but not superstrong and there are no measurable cardinals in the half-open interval $(\delta, \lambda]{ }^{\prime \prime}, \dot{\mathbb{R}}^{1}$ is a term for the partial ordering adding a non-reflecting stationary set of ordinals of cofinality $\omega$ to $\delta$, and the first ordinal at which $\dot{\mathbb{R}}^{2}$ is forced to do nontrivial forcing is well beyond $\lambda$. Next, working in $M$, we consider the "term forcing" partial ordering $\mathbb{T}$ (see [6] for the first published account of term forcing or [5, Section 1.2 .5 , page 8 ]; the notion is originally due to Laver) associated with $\dot{\mathbb{R}}^{2}$. This is given by $\tau \in \mathbb{T}$ iff $\tau$ is a term in the forcing language with respect to $\mathbb{P}_{\delta} * \dot{\mathbb{R}}^{1}$ and $\Vdash_{\mathbb{P}_{\delta} * \mathbb{R}^{1}}$ " $\tau \in \dot{\mathbb{R}}^{2}$ ", with ordering $\tau_{1} \geq \tau_{0}$ iff $\vdash_{\mathbb{P}_{\delta} * \dot{\mathbb{R}}^{1}}$ " $\tau_{1} \geq \tau_{0}$ ". As in the proof of $[9$, Theorem 4.1$]$ (see also the proof of $[3$, Lemma 2.3]), we may assume without loss of generality that $M \vDash "|\mathbb{T}|=j(\delta) "$. Since $\Vdash_{\mathbb{P}_{\delta} * \mathbb{R}^{1}}$ " $\dot{\mathbb{R}}^{2}$ is $\prec \delta^{+}$-strategically closed", it can easily be verified that $\mathbb{T}$ is also $\prec \delta^{+}$-strategically closed in $M$, and, since $M^{\delta} \subseteq M$, in $V$ as well. In addition, because $\Vdash_{\mathbb{P}_{\delta} * \mathbb{R}^{1}}$ " $\dot{\mathbb{R}}^{2}$ is $\lambda$-strategically closed", $M \vDash " \mathbb{T}$ is $\lambda$-strategically closed and hence is also $(\lambda, \infty)$-distributive".

Let $X=\{j(f)(\delta, \lambda): f \in V\}$. As in the proof of [9, Theorem 4.1], $X \prec M$ and $X$ is isomorphic to the ultrapower of $V$ via the measure $\mu$ on $\delta \times \delta$ given by $A \in \mu$ iff $\langle\delta, \lambda\rangle \in j(A)$. If we let $j_{0}: V \rightarrow M_{0}$ be the ultrapower embedding by $\mu$, then $j=j_{1} \circ j_{0}$, where $j_{0}=\pi \circ j$ for $\pi$ the Mostowski collapse of $X$ to $M_{0}, j_{1}: M_{0} \rightarrow M$ has critical point greater than $\delta$, and $j_{1}=\pi^{-1}$. The situation is given by the commutative diagram

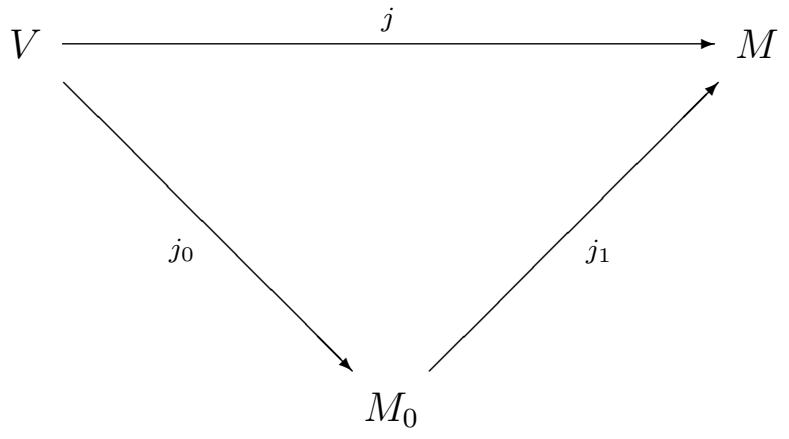

If $\mathbb{T}_{0}=\pi(\mathbb{T})$ and $\lambda_{0}=\pi(\lambda)$, then $\mathbb{T}_{0}$ has size $j_{0}(\delta)$ in $M_{0}$. Further, $\mathbb{T}_{0}$ is $\prec \delta^{+}$-strategically

\footnotetext{
${ }^{4}$ It is because $M \vDash$ " $\lambda$ is an inaccessible cardinal which is not a limit of inaccessible cardinals" (which of course follows since $\left.V_{\lambda} \subseteq M\right)$ that we may infer that $M \vDash$ "There are no measurable cardinals in the half-open interval $(\delta, \lambda] "$
} 
closed in $M_{0}$ and, since $\left(M_{0}\right)^{\delta} \subseteq V$, in $V$ as well. The argument used in the construction of $G_{1}$ may therefore be employed to construct in $V$ an $M_{0}$-generic object $G_{2}^{*}$ for $\mathbb{T}_{0}$.

We claim that the filter $G_{2}^{* *} \subseteq \mathbb{T}$ generated by $j_{1}^{\prime \prime} G_{2}^{*}$ is $M$-generic over $\mathbb{T}$. To see this, let $D \in M$ be a dense open subset of $\mathbb{T}$. Hence, $D=j(f)(s)$ for some $f: V_{\delta} \rightarrow V$, where $f \in V$ and $s \in V_{\lambda}$. It therefore follows that $D=j_{1}(r)(s)$, where $r=j_{0}(f) \uparrow\left(V_{\lambda_{0}}\right)^{M_{0}}$. We may assume that $r(t)$ is a dense open subset of $\mathbb{T}_{0}$ for every $t \in\left(V_{\lambda_{0}}\right)^{M_{0}}$. Consequently, since $M_{0} \vDash "\left|V_{\lambda_{0}}\right|=\lambda_{0}$ and $\mathbb{T}_{0}$ is $\left(\lambda_{0}, \infty\right)$-distributive", $\bar{D}=\bigcap_{t \in\left(V_{\lambda_{0}}\right)^{M_{0}}} r(t)$ is a dense open subset of $\mathbb{T}_{0}$ in $M_{0}$. It then follows that $\bar{D} \cap G_{2}^{*} \neq \emptyset$, which further implies that $j_{1}(\bar{D}) \cap G_{2}^{* *} \neq \emptyset$. As $j_{1}(\bar{D}) \subseteq D, D \cap G_{2}^{* *} \neq \emptyset$, i.e., $G_{2}^{* *}$ is $M$-generic over $\mathbb{T}$.

Note now that since $N$ is the ultrapower of $M$ via the normal measure $\mathcal{U} \in M$ over $\delta$, [5, Fact 2 of Section 1.2.2] tells us that $k^{\prime \prime} G_{2}^{* *}$ generates an $N$-generic object $G_{2}^{* * *}$ over $k(\mathbb{T})$. By elementarity, $k(\mathbb{T})$ is the term forcing partial ordering defined in $N$ with respect to $k\left(j\left(\mathbb{P}_{\delta}\right)_{\delta+1}\right)=\mathbb{P}_{\delta} * \dot{\mathbb{Q}}^{1}$. Therefore, since $i\left(\mathbb{P}_{\delta}\right)=k\left(j\left(\mathbb{P}_{\delta}\right)\right)=\mathbb{P}_{\delta} * \dot{\mathbb{Q}}^{1} * \dot{\mathbb{Q}}^{2}, G_{2}^{* * *}$ is $N$-generic over $k(\mathbb{T})$, and $G_{0} * G_{1}$ is $N$-generic over $k\left(\mathbb{P}_{\delta} * \dot{\mathbb{R}}^{1}\right)$, [5, Fact 1 of Section 1.2.5] (see also [6]) tells us that $G_{2}=\left\{i_{G_{0} * G_{1}}(\tau): \tau \in G_{2}^{* * *}\right\}$ is $N\left[G_{0}\right]\left[G_{1}\right]$-generic over $\mathbb{Q}^{2}$. It now follows that $i$ lifts in $V\left[G_{0}\right]$ to $i: V\left[G_{0}\right] \rightarrow N\left[G_{0}\right]\left[G_{1}\right]\left[G_{2}\right]$, i.e., $V\left[G_{0}\right] \vDash$ " $\delta$ is $\lambda$ tall". This completes the proof of Lemma 2.4.

Lemma 2.5 $V^{\mathbb{P}} \vDash$ "Every measurable cardinal $\delta \neq \kappa$ is $\lambda_{\delta}$ tall, for some inaccessible cardinal $\lambda_{\delta}>\rho_{\delta} "$.

Proof: As in the proof of Lemma 2.4, we may assume without loss of generality that $\delta<\kappa$. In addition, as in the proof of Lemma 2.3, $\mathbb{P}$ is mild with respect to $\delta$. Therefore, by the factorization of $\mathbb{P}\left(\right.$ as $\left.\mathbb{P}^{\prime} * \dot{\mathbb{P}}^{\prime \prime}\right)$ given in Lemma 2.2 and Theorem 3 , if $V^{\mathbb{P}} \vDash$ " $\delta$ is $\alpha$ strongly compact", then $V \vDash ~ " ~ \delta$ is $\alpha$ strongly compact" as well. Consequently, since $\delta<\kappa,\left(\rho_{\delta}\right)^{V}$ is defined, and $\left(\rho_{\delta}\right)^{V^{\mathbb{P}}} \leq\left(\rho_{\delta}\right)^{V}<\left(\sigma_{\delta}\right)^{V}$. By Lemma 2.4 and its proof, $V^{\mathbb{P}} \vDash$ "There is an inaccessible cardinal $\lambda_{\delta}>\rho_{\delta}$ such that $\delta$ is $\lambda_{\delta}$ tall". This completes the proof of Lemma 2.5. 
Since $V^{\mathbb{P}} \vDash$ "Level by level equivalence holds and there are no measurable cardinals above $\kappa$ ", $V^{\mathbb{P}} \vDash " \kappa$ is the only strongly compact cardinal". This fact, together with Lemmas $2.1-2.5$ and the intervening remarks, complete the proof of Theorem 1.

Having completed the proof of Theorem 1, we turn now to the proof of Theorem 2.

Proof: Suppose $V \vDash$ "ZFC $+\mathrm{GCH}+\kappa<\lambda$ are such that $\kappa$ is $\lambda$ supercompact and $\lambda$ is the least inaccessible cardinal above $\kappa+$ Level by level equivalence holds". Without loss of generality (but with a slight abuse of notation), we assume that $\kappa$ is the least cardinal which is $\gamma$ supercompact for some inaccessible cardinal $\gamma>\kappa$. This immediately implies that no cardinal $\delta<\kappa$ is either supercompact or strongly compact up to a measurable cardinal (and in fact, that no cardinal $\delta<\kappa$ is either supercompact or strongly compact up to the second inaccessible cardinal above it).

Let $B=\{\delta<\kappa$ : Either $\delta$ is a non-superstrong measurable cardinal or $\delta$ is not supercompact up to the least inaccessible cardinal above it\}. The partial ordering $\mathbb{P}$ used in the proof of Theorem 2 is then defined as the reverse Easton iteration of length $\kappa$ which begins by adding a Cohen subset of $\omega$ and then does nontrivial forcing only at stages $\delta \in B$, where it adds a non-reflecting stationary set of ordinals of cofinality $\omega$ to $\delta$. Standard arguments once again show that $V^{\mathbb{P}} \vDash G C H$ and that $V$ and $V^{\mathbb{P}}$ have the same cardinals and cofinalities.

Lemma $2.6 V^{\mathbb{P}} \vDash$ " $\kappa$ is $\lambda$ supercompact".

Proof: Let $j: V \rightarrow M$ be an elementary embedding witnessing the $\lambda$ supercompactness of $\kappa$ generated by a supercompact ultrafilter over $P_{\kappa}(\lambda)$. As in the proof of Lemma 2.1, note that $M \vDash$ " $\kappa$ is superstrong". Therefore, by the definition of $\mathbb{P}$ and the fact that $M \vDash$ " $\lambda$ is the least inaccessible cardinal greater than $\kappa$ and $\kappa$ is supercompact up to $\lambda ", j(\mathbb{P})=\mathbb{P} * \dot{\mathbb{Q}}$, where the first ordinal at which $\dot{\mathbb{Q}}$ is forced to do nontrivial forcing is well above $\lambda$. The same arguments as used in the proof of Lemma 2.1 now show that $j$ lifts in $V^{\mathbb{P}}$ to a $\lambda$ supercompactness embedding $j: V^{\mathbb{P}} \rightarrow M^{j(\mathbb{P})}$. This completes the proof of Lemma 2.6. 
Using Lemma 2.6 and reflection, we may now let $\kappa_{0}<\kappa$ be the smallest cardinal such that $V^{\mathbb{P}} \vDash " \kappa_{0}$ is supercompact up to some inaccessible cardinal $\lambda_{0}$ ". Without loss of generality, we assume in addition that $V^{\mathbb{P}} \vDash$ " $\lambda_{0}$ is the least inaccessible cardinal above $\kappa_{0}$ ".

Lemma 2.7 $V^{\mathbb{P}} \vDash$ "If $\delta<\kappa_{0}$ is measurable, then $\delta$ is strongly compact up to the least inaccessible cardinal above it".

Proof: We may assume that $V \vDash " \delta$ is both superstrong and supercompact up to the least inaccessible cardinal above it". (If this is not the case, then by the definition of $\mathbb{P}, V^{\mathbb{P}} \vDash$ " $\delta$ contains a non-reflecting stationary set of ordinals of cofinality $\omega "$.) Since $\left|\mathbb{P}_{\delta}\right|=\delta$, this means that we may write $\mathbb{P}=\mathbb{P}_{\delta} * \dot{\mathbb{P}}^{\delta}$, where $\Vdash_{\mathbb{P}_{\delta}}$ "Forcing with $\dot{\mathbb{P}}^{\delta}$ adds no bounded subsets to the least measurable cardinal above $\delta$ ". In particular, $\Vdash_{\mathbb{P}_{\delta}}$ "Forcing with $\dot{\mathbb{P}}^{\delta}$ adds no subsets to the least inaccessible cardinal above $\delta "$. Thus, in order to prove Lemma 2.7, it suffices to show that $V^{\mathbb{P}_{\delta}} \vDash " \delta$ is strongly compact up to the least inaccessible cardinal above it".

To do this, let $\lambda>\delta$ be a regular cardinal below the least $V$-inaccessible cardinal above $\delta$ (which, by the factorization of $\mathbb{P}$ given in the preceding paragraph, is the same as the least $V^{\mathbb{P}}$-inaccessible or

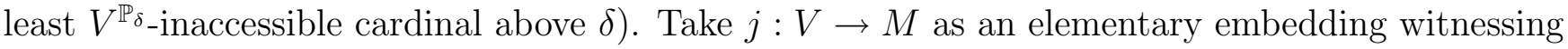
the $\lambda$ supercompactness of $\delta$ generated by a supercompact ultrafilter over $P_{\delta}(\lambda)$ such that $M \vDash " \delta$ is not $\lambda$ supercompact". Note that $M \vDash$ " $\lambda$ is below the least inaccessible cardinal above it". Therefore, by the definition of $\mathbb{P}, j\left(\mathbb{P}_{\delta}\right)=\mathbb{P}_{\delta} * \dot{\mathbb{R}}^{1} * \dot{\mathbb{R}}^{2}$, where $\dot{\mathbb{R}}^{1}$ is a term for the partial ordering adding a non-reflecting stationary set of ordinals of cofinality $\omega$ to $\delta$, and the first ordinal at which $\dot{\mathbb{R}}^{2}$ is forced to do nontrivial forcing is well above $\lambda$. The same argument as found in the proofs of $\left[2\right.$, Lemma 2.4] and [3, Lemma 2.3] now shows that $V^{\mathbb{P}_{\delta}} \vDash$ " $\delta$ is $\lambda$ strongly compact". 5 Since $\lambda$

\footnotetext{
${ }^{5}$ An outline of this argument is as follows. Let $k: M \rightarrow N$ be an elementary embedding generated by a normal measure $\mathcal{U} \in M$ having trivial Mitchell rank. The elementary embedding $i=k \circ j$ witnesses the $\lambda$ strong compactness of $\delta$ in $V$. As in the proof of Lemma 2.4, this embedding lifts in $V^{\mathbb{P}_{\delta}}$ to an elementary embedding $i: V^{\mathbb{P}_{\delta}} \rightarrow N^{i\left(\mathbb{P}_{\delta}\right)}$ witnessing the $\lambda$ strong compactness of $\delta$. This is shown by writing $i\left(\mathbb{P}_{\delta}\right)=\mathbb{P}_{\delta} * \dot{\mathbb{Q}}^{1} * \dot{\mathbb{Q}}^{2}$, taking $G_{0}$ to be $V$-generic over $\mathbb{P}_{\delta}$, and building in $V\left[G_{0}\right]$ generic objects $G_{1}$ and $G_{2}$ for $\mathbb{Q}^{1}$ and $\mathbb{Q}^{2}$ respectively. The construction of $G_{1}$ is the same as that found for the generic object $G_{1}$ in the proof of Lemma 2.4. The construction of $G_{2}$ first requires building an $M$-generic object $G_{2}^{* *}$ for the term forcing partial ordering $\mathbb{T}$ associated with $\dot{\mathbb{R}}^{2}$ and defined in $M$ with respect to $\mathbb{P}_{\delta} * \dot{\mathbb{R}}^{1}$. The current $G_{2}^{* *}$ is built using the fact that since $M^{\lambda} \subseteq M, \mathbb{T}$ is $\prec \lambda$-strategically closed in both $M$ and $V$, which means that the diagonalization argument employed in the construction of $G_{1}$ may be applied in this situation as well. $k^{\prime \prime} G_{2}^{* *}$ now generates an $N$-generic object $G_{2}^{* * *}$ for $k(\mathbb{T})$ and an $N\left[G_{0}\right]\left[G_{1}\right]$-generic object $G_{2}$ for $\mathbb{Q}^{2}$ as in the proof of Lemma 2.4. This means that $i$ lifts in $V\left[G_{0}\right]$ to $i: V\left[G_{0}\right] \rightarrow N\left[G_{0}\right]\left[G_{1}\right]\left[G_{2}\right]$.
} 
was an arbitrary regular cardinal below the least $V^{\mathbb{P}_{\delta}}$-inaccessible cardinal above $\delta$, the proof of Lemma 2.7 has consequently been completed.

Lemma 2.8 $V^{\mathbb{P}} \vDash$ "Every measurable cardinal $\delta<\kappa_{0}$ witnesses level by level inequivalence".

Proof: By the choice of $\kappa_{0}$, if $V^{\mathbb{P}} \vDash " \delta<\kappa_{0}$ is measurable", then $V^{\mathbb{P}} \vDash$ " $\theta_{\delta}$ is below the least inaccessible cardinal above it". However, by Lemma $2.7, V^{\mathbb{P}} \vDash$ " $\delta$ is strongly compact up to the least inaccessible cardinal above it". This completes the proof of Lemma 2.8.

Let $\bar{V}=\left(V_{\lambda_{0}}\right)^{V^{\mathbb{P}}}$. By the definition of $\mathbb{P}, \bar{V} \vDash$ "There are unboundedly in $\kappa_{0}$ many cardinals $\delta<\kappa_{0}$ containing non-reflecting stationary sets of ordinals of cofinality $\omega "$. Therefore, by [12, Theorem 4.8] and the succeeding remarks, $\bar{V} \vDash$ "No cardinal $\delta<\kappa_{0}$ is strongly compact". In addition, using the factorization of $\mathbb{P}\left(\right.$ as $\left.\mathbb{P}_{\delta} * \dot{\mathbb{P}}^{\delta}\right)$ given in Lemma 2.7 , the same arguments as found in the proofs of Lemmas 2.4 and 2.5 show that $V^{\mathbb{P}} \vDash$ "Every measurable cardinal $\delta<\kappa_{0}$ is $\lambda_{\delta}$ tall, for some inaccessible cardinal $\lambda_{\delta}>\rho_{\delta}$ ". These facts, together with Lemmas $2.6-2.8$, the intervening remarks, and the fact that $\bar{V} \vDash$ "No cardinal above $\kappa_{0}$ is inaccessible", complete the proof of Theorem 2 .

Since $\left|\mathbb{P}_{\delta}\right|=\delta$ in the above proofs, the results of [11] imply that for any ordinal $\gamma$ in the open interval $\left(\delta, \sigma_{\delta}\right)$, the least weakly compact cardinal above $\gamma$, the least Ramsey cardinal above $\gamma$, the least Ramsey limit of Ramsey cardinals above $\gamma$, or in general, any large cardinal provably in the open interval $\left(\gamma, \sigma_{\delta}\right)$ for which the results of [11] hold are the same in $V$ and $V^{\mathbb{P}_{\delta}}$. It is for this reason, together with the fact that $\vdash_{\mathbb{P}_{\delta}}$ " $\dot{\mathbb{P}}^{\delta}$ is $\prec\left(\sigma_{\delta}\right)^{V}$-strategically closed and $\left(\sigma_{\delta}\right)^{V}=\left(\sigma_{\delta}\right)^{V^{\mathbb{P}} \delta}$, that the witnessing models for Theorems 1 and 2 have each non-supercompact measurable cardinal $\delta$ exhibit $\lambda_{\delta}$ tallness for $\lambda_{\delta}$ the least weakly compact cardinal above $\rho_{\delta}$, the least Ramsey cardinal above $\rho_{\delta}$, the least Ramsey limit of Ramsey cardinals above $\rho_{\delta}$, etc. On the other hand, our 
methods of proof seem to require ground models with a severely restricted large cardinal structure (although the same proof techniques will allow, e.g., the definition of $\lambda$ in Theorem 2 to be changed to the least weakly compact cardinal above $\kappa$, thereby giving a model witnessing the conclusions of Theorem 2 with a slightly richer large cardinal structure). We conclude by asking if it is possible to remove these restrictions, and obtain results analogous to those of this paper in which the large cardinal structure of the universe can be arbitrary.

\section{References}

[1] A. Apter, "On Level by Level Equivalence and Inequivalence between Strong Compactness and Supercompactness", Fundamenta Mathematicae 171, 2002, 77-92.

[2] A. Apter, J. Cummings, "Identity Crises and Strong Compactness II: Strong Cardinals", Archive for Mathematical Logic 40, 2001, 25-38.

[3] A. Apter, J. D. Hamkins, "Exactly Controlling the Non-Supercompact Strongly Compact Cardinals", Journal of Symbolic Logic 68, 2003, 669-688.

[4] A. Apter, S. Shelah, "On the Strong Equality between Supercompactness and Strong Compactness", Transactions of the American Mathematical Society 349, 1997, 103-128.

[5] J. Cummings, "A Model in which GCH Holds at Successors but Fails at Limits", Transactions of the American Mathematical Society 329, 1992, 1-39.

[6] M. Foreman, "More Saturated Ideals", in: Cabal Seminar 79-81, Lecture Notes in Mathematics 1019, Springer-Verlag, Berlin and New York, 1983, 1-27.

[7] J. D. Hamkins, "Gap Forcing”, Israel Journal of Mathematics 125, 2001, 237-252.

[8] J. D. Hamkins, "Gap Forcing: Generalizing the Lévy-Solovay Theorem", Bulletin of Symbolic Logic 5, 1999, 264-272.

[9] J. D. Hamkins, "Tall Cardinals", Mathematical Logic Quarterly 55, 2009, 68-86. 
[10] T. Jech, Set Theory: The Third Millennium Edition, Revised and Expanded, Springer-Verlag, Berlin and New York, 2003.

[11] A. Lévy, R. Solovay, "Measurable Cardinals and the Continuum Hypothesis", Israel Journal of Mathematics 5, 1967, 234-248.

[12] R. Solovay, W. Reinhardt, A. Kanamori, "Strong Axioms of Infinity and Elementary Embeddings", Annals of Mathematical Logic 13, 1978, 73-116. 